\title{
Is perioperative brachytherapy effective in carcinoma of the tongue?
}

\author{
Miguel Santos, MD!', José Luis Guinot, MD, PhD', Maribel Tortajada, MD', Juan Bosco Vendrell, MD, PhD², \\ Celia López, MD, PhD³, Alonso La Rosa, MD!, Beatriz Quiles, MD!, Marisa Chust, MD!, Alba Montaner, MSc ${ }^{4}$ \\ Leo Arribas, MD, PhD 1,5 \\ 'Department of Radiation Oncology, Fundación Instituto Valenciano de Oncología (FIVO), Valencia, Spain, 2Department of Otolaryngology, \\ Fundación Instituto Valenciano de Oncología (FIVO), Valencia, Spain, ${ }^{3}$ Department of Otolaryngology, Head \& Neck Surgery. \\ Hospital Universitario Dr. Peset, Valencia, Spain, ${ }^{4}$ Department of Radiation Physics and Radiological Protection, Fundación Instituto Valenciano \\ de Oncología (FIVO), Valencia, Spain, ${ }^{5}$ Universidad Católica de Valencia San Vicente Mártir, Spain
}

\begin{abstract}
Purpose: To analyze the results of patients treated with perioperative interstitial brachytherapy (ISBT) in tongue carcinoma (TC).

Material and methods: From April 2009 to May 2015, 43 squamous cell carcinoma consecutive patients diagnosed with TC were treated with limited partial glossectomy and perioperative ISBT, using high-dose-rate (HDR). Twentyseven patients were treated by brachytherapy (BT), and sixteen received BT as a complement to subsequent external beam radiotherapy (EBRT) after results of lymph node dissection. Median age was 66 years. Distribution by stage, included 10 patients stage I, 14 stage II, 10 stage III, and 9 stage IV. Eighteen patients had negative margins, nineteen margin involvement, and in six cases, the margin was $<5 \mathrm{~mm}$.

Results: With a median follow-up of 54 months, LC at 3 and 5 years was $87 \%$ and $84 \%$, respectively. LC was $95 \%$ at five years in patients with clear margins, and 75\% with involved margins. LC in N0 patients treated with BT was 83\% at 5 years, and in patients $\mathrm{N}+$ with posterior EBRT treatment, LC was 86\%. By tumor size, we found one local relapse in 13 cases T1, in 5 of 27 patients T2 was found, and no local relapse T3 with LC of $87 \%$, 70\%, and $100 \%$ respectively at five years. Regional control (RC) was $81 \%$ at 3 and 5 years. We found a metastasis-free survival of $91 \%$ at 3- and 5-year. Twenty-three patients have died, 11 of them due to other causes, with overall survival of $56 \%$ at three years and $53 \%$ at five years.

Conclusions: Combined treatment with conservative surgery and ISBT shows similar results to radical surgery or RT alone, allowing a more patient-tailored approach, with good organ function preservation and cosmetic outcomes.

Key words: brachytherapy, high-dose-rate, tongue carcinoma, perioperative.

\section{Purpose}

Surgery is considered the standard of care for earlystage squamous cell carcinoma of the oral tongue [1, 2]. However, radiotherapy is deemed as a possible option in some situations and in certain patient profiles, providing good local control of the disease.

The presence of risk factors, such as close or positive margins, perineural invasion, and/or extracapsular involvement, reinforces the indication of adjuvant radiation $[3,4]$.

Brachytherapy can be used as part of the management in different contexts. First, as an exclusive treatment; second, complementary to surgery; third, as a boost in combination with external beam radiation (EBRT).

High-dose-rate brachytherapy (HDR-BT) is a technique that allows dose optimization and organ protection due to a more localized and controlled radiation delivery in comparison with EBRT. This technique can be useful for dose escalation to the tumor bed in this scenario (post-operative tongue radiation) [5, 6]. It also reduces the chances of ending up with an organ dysfunction, such as dysphagia (swallowing) or communication disabilities (phonatory abnormalities), resulting in a direct impact on quality of life [7].

Perioperative BT has the advantage of tumor bed exposing; therefore, it can be better covered with a smaller implant, it is performed during surgery avoiding a second anesthesia, and radiation can be delivered earlier.

After analyzing 50 patients with tongue carcinoma treated with HDR-BT, Guinot et al. reported $100 \%$ local control in 14 patients, in whom brachytherapy was delivered perioperatively during a 5 years of follow-up [8]. Moreover, a retrospective study that combined EBRT and HDR-BT boost in locally advanced tongue carcinoma,

Address for correspondence: Miguel Santos Olias, Department of Radiation Oncology, Fundación Instituto Received: 14.10 .2021 Valenciano de Oncología (FIVO), C/Beltrán Baguena 8, Valencia, 46008, Spain, phone: +34-625-266-055, 
postulated this approach as a good option in terms of good local control (LC) and cause-specific survival (CSS), providing a favorable adverse effects profile [9].

The aim of this retrospective study was to assess the efficacy of perioperative brachytherapy in tongue carcinoma to perform a smaller volume surgery, and to achieve better preservation of the function of mobile tongue.

\section{Material and methods}

All patients treated with perioperative BT at our department between April 2009 and May 2015 were recorded. The BT implant requires a collaborative multidisciplinary team approach involving a radiation oncologist, nurse and physicist, a head and neck surgeon, and an anesthetist. This treatment is considered standard as an alternative to post-operative brachytherapy. It was authorized by the tumor board, and each patient signed an informed consent. The selection criteria were all cases with mobile tongue carcinoma between 1.5 and $5 \mathrm{~cm}$ in computed tomography (CT) or magnetic resonance (MR) imaging, susceptible to partial tongue glossectomy without requiring a graft, and with an intention to avoid hemiglossectomy.

Perioperative implant was performed to improve the results of the same surgical procedure, and to prevent application of second general anesthesia. Surgical intervention was a partial glossectomy, smaller than an hemiglossectomy, with the aim of removing the gross tumor with an adequate margin as well as preserving a better tongue function. If a close or involved margin remains, BT would begin after 3 or 4 days, and the final dose will be higher. No re-resection was done to achieve more adequate margins.

Implantation procedure and general definition process in our institution have been previously described [8]. Once the neck dissection and partial glossectomy were performed, an array of parallel rigid needles were inserted through the submandibular area. Needles were substituted by plastic tubes, with a button on the top and fixed with another button on the skin. The implant was done with precise knowledge of where the tumor was located; hence, a smaller implant was required with a range of $5-9$ tubes aiming at achieving a spacing of $10 \mathrm{~mm}$ between them in two planes [8]. The procedure prolonged the surgical time from 20 to 30 minutes.

A CT planning scan was done three days after the surgery with $3 \mathrm{~mm}$ slices. Clinical target volume (CTV) was defined around the plastic tubes with a margin of 2-3 mm because tumor was no longer visible. The mandible was drawn as an organ of risk. A modified Paris system was used to calculate the isodoses with the Nucletron ${ }^{\circledR}$ (an ELEKTA company) treatment planning system (TPS). The prescription dose was to $90 \%$ isodose of the basal dose, with a manual adjustment of the isodoses curves to decrease the dose to $0.1 \mathrm{cc}$ of the mandible under the prescription dose. We tried to keep a DNR (dose-non-uniformity ratio, $\mathrm{V}_{150} / \mathrm{V}_{100}$ ) under 0.35 [8] (Figure 1 ).

We started to treat on the third or fourth day after surgery with a standard dose of 4 Gy per fraction twice a day, six hours apart. When five sessions were administered, we obtained a pathologist's report and the total dose was decided. If lymph node involvement was confirmed, BT was deemed completed, and the plastic tubes were withdrawn, allowing for EBRT to be started within 2 to 4 weeks after BT ending, to ensure complete treatment to the oral cavity and cervical lymph nodes. Twenty-seven patients received BT exclusively, and sixteen received additional EBRT after the results of lymph node dissections. The median total dose was 40-44 Gy when HDR-BT was exclusive, and 18-20 Gy (biological equivalent dose [BED], 25-28 Gy) when HDR-BT was administered in combination with EBRT. A total dose of $44 \mathrm{~Gy}$ in 11 fractions of $4 \mathrm{~Gy}$ (BED, 61.6 Gy) was administered in twenty-five patients, including nineteen patients with positive margins, and six cases, in which surgical margin was considered as 'close', defined as less than $5 \mathrm{~mm}$ margin from the tumor to the resection margin.

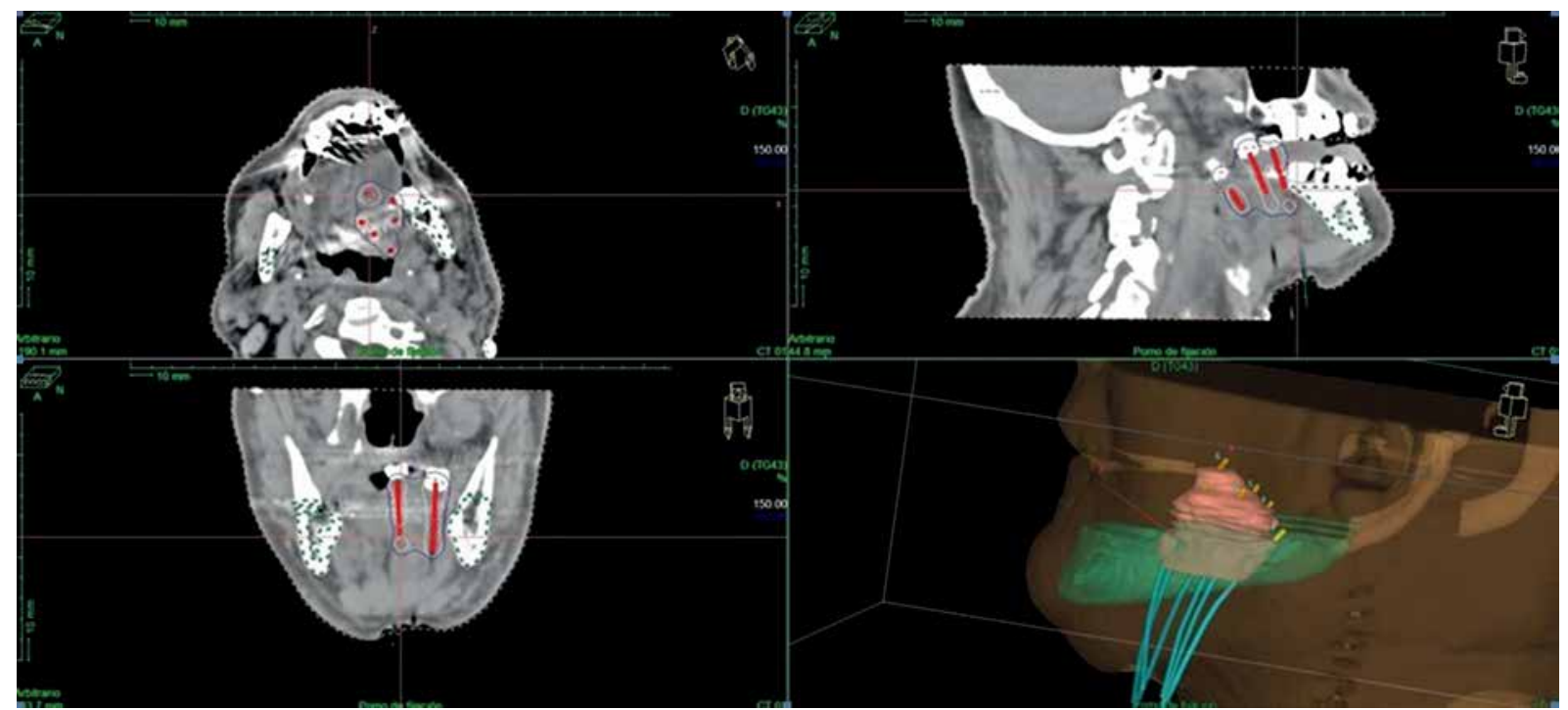

Fig. 1. CT planning scan, with manual adjustment of isodoses curves to decrease the dose to $0.1 \mathrm{cc}$ of the mandible under prescription dose, keeping a DNR $\left(\mathrm{V}_{150} / \mathrm{V}_{100}\right)$ under 0.35 (in this case, 0.27 ) 
For the other 18 patients, with free or negative surgical margins, we still considered that BT was required because most of them presented various risk factors, such as deep infiltration $>5 \mathrm{~mm}$, tumor size $>3 \mathrm{~cm}$, or perineural involvement, with surgery done as very conservative procedure; therefore, a total dose of $40 \mathrm{~Gy}$ in 10 fractions (BED, 56 Gy) was delivered.

Doses of EBRT to both necks were in the range of 60-66 Gy to the involved area, and 54-60 Gy to the rest of the neck. Oral cavity was treated up to 54-56 Gy, including partial glossectomy. Chemotherapy concomitant with EBRT was applied in patients with more than one involved lymph node, but not in cases with positive margin.

For statistical analysis regarding LC, regional control (RC), overall survival (OS), CSS, and disease-free survival (DFS) rates, corrected actuarial survivals, were calculated using Kaplan-Meier method. Local recurrences were considered as tumor growth in the same area or close to the primary tumor, confirmed by biopsy. Regional recurrences were considered as any regional lymph node growth in the cervical area.

\section{Results}

\section{Patients' characteristics}

Forty-three patients diagnosed with carcinoma of the tongue were treated with perioperative ISBT. There were eighteen males and twenty-five females. The mean age was 63 years (range, 25-93 years). All tumors were histologically confirmed, indicating invasive squamous carcinoma.

The distribution by stages included 10 patients stage I, 14 stage II, 10 stage III, and 9 stage IV. The pathological distribution of tumor size was thirteen patients T1, twenty-seven T2, and three patients T3. Depth of invasion was not considered at the time of staging. Sixteen patients $(37 \%)$ received a subsequent EBRT, after the result of lymph node dissection.

\section{Local control}

With a median follow-up of 54 months (range, 3-163 months), LC at 3 and 5 years was 87 and $84 \%$, respectively, for all cases (Figure 2). LC in N0 patients who received treatment with exclusive BT was $83 \%$ at 5 years, and in patients with lymph node involvement and subsequent EBRT treatment, LC was $86 \%$.

By stages, we achieved a LC of $85 \%$ in stage I, $78 \%$ in II, $76 \%$ in III, and $100 \%$ in stage IV. By tumor size, there was 1 local relapse in the thirteen T1patients, 5 relapses in the twenty-seven $\mathrm{T} 2$ patients, and no local relapse in three T3 patients, resulting in a LC of $87 \%, 70 \%$, and $100 \%$, respectively, at 5 years.

Regarding surgical margins, of the 19 patients who presented involved margins, there were 3 local relapses, and LC was $80 \%$ at 3 and 5 years. For the 18 patients with negative margins, there was 1 local relapse and LC of $95 \%$, and finally, there were 6 patients with close margins with 2 local relapses, achieving a LC of $83 \%$ and $41 \%$ at 3 and 5 years, respectively (Figure 3 ). If we analyzed together the 25 patients with involved and close margins, there were 5 local relapses, and LC of $75 \%$ at 3 and 5 years (Figure 4).

\section{Regional and distant control}

Regional control was $81 \%$ at 3 and 5 years. In the sixteen patients with nodal involvement, there were two events, with RC at 5 years of $84 \%$. In twenty-seven patients free of nodal disease, there were five nodal recurrences, with a RC of $79 \%$. Three patients presented distant

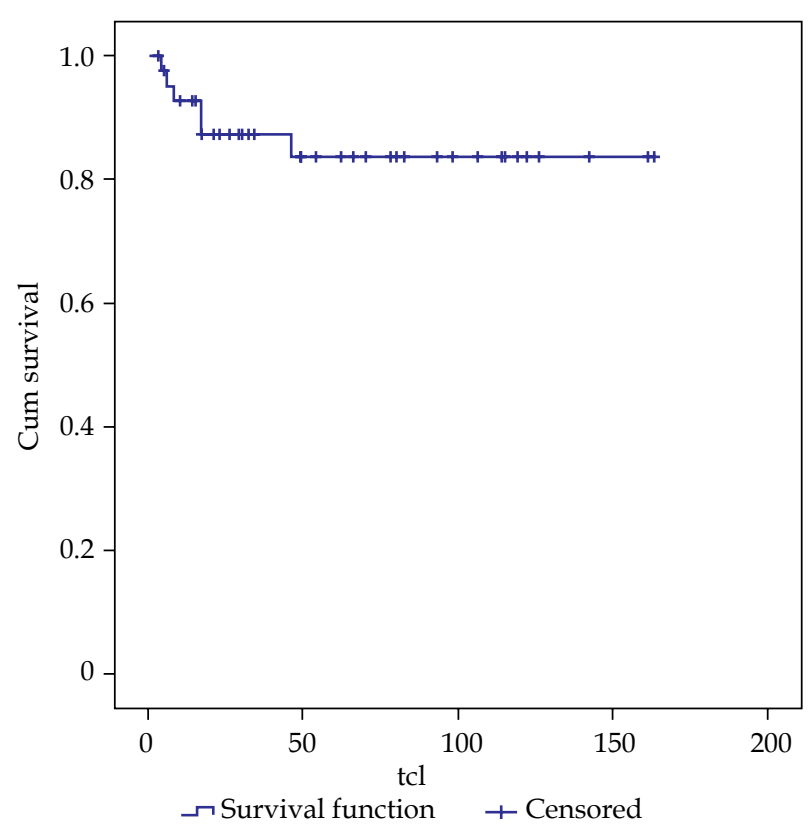

Fig. 2. Kaplan-Meier local control probability

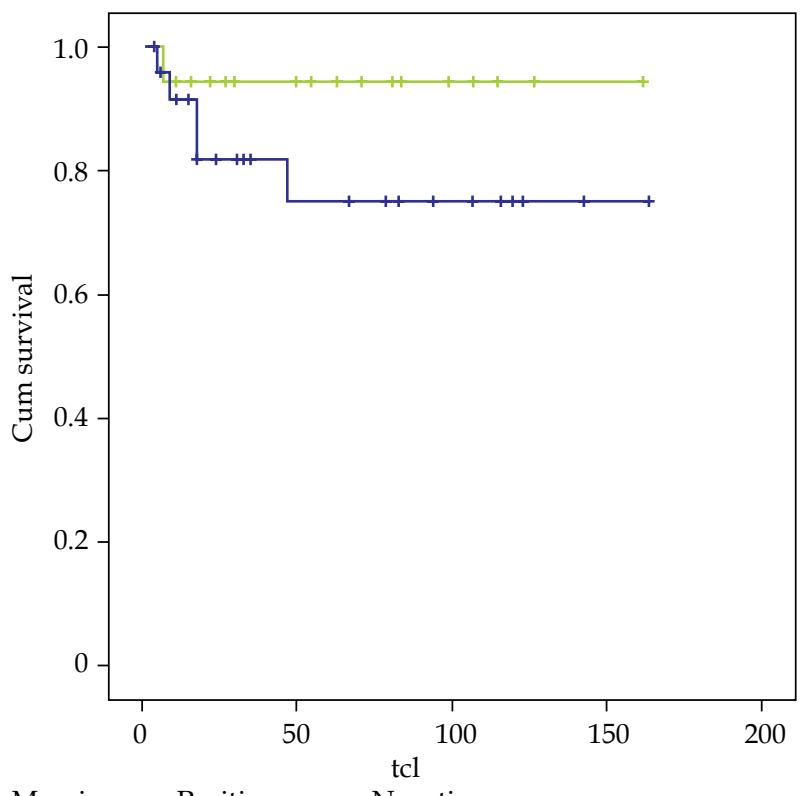

Margins $\neg$ Positive $\neg$ Negative

+ Positive censored + Negative censored

Fig. 3. Local control probability according to margins status, negative or positive 


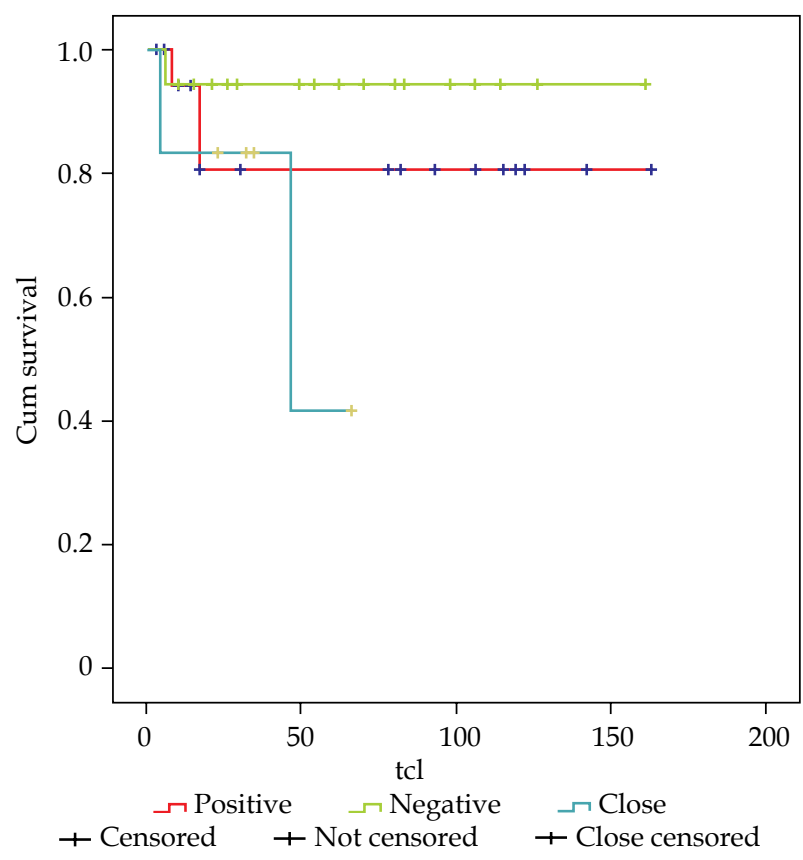

Fig. 4. Local control probability according to margins status: positive, negative, or close

metastases, thus achieving a 3- and 5-year metastasis-free survival of $91 \%$ for the entire cohort.

\section{Overall and cancer-specific survival}

Twenty-three patients died, out of whom, eleven of non-cancer specific causes, with an overall survival of $56 \%$ at 3 years, and $53 \%$ at 60 months. The cancer-specific survival achieved at 3 and 5 years was $88 \%$.

\section{Complications}

Acute complications were similar to those described with low-dose-rate (LDR) BT. During removal of the tubes, $16 \%$ cases of small bleeding were treated with compressive measures. Mucositis in the implanted area was always present, and lasted for 4-6 weeks until complete recovery. Acute mucositis and odynophagia were related in almost all patients who were treated with EBRT. Late complications were limited to one case of mandibular bone necrosis $(2 \%)$, resolved with medical management (G2), 4 temporary soft tissue ulcer G2 (9.3\%), and two cases of chronic pain G2. No case of G3 toxicity was reported (CTCAE v.5.0).

\section{Discussion}

\section{Surgery}

Oral cavity cancers, in particular tongue cancers, are generally treated with primary surgery whenever possible. Extensive surgery, such as hemiglossectomy, offers good rates of LC, but can leave permanent impairments in function. Radiation therapy and systemic treatment can be part of management in some situations.

Post-operative margin status is of important value in deciding adjuvant treatment and predicting prognosis.
The removal of microscopic or sub-clinical foci of malignancy is essential and critical to achieve successful local control of the disease [10], since the inability to achieve clear surgical margins increases the risk of local recurrence and subsequently, reduces the survival rates [11-16]. The surgical margin is considered clear when the distance is $>5 \mathrm{~mm}$, close when it is $1-5 \mathrm{~mm}$, and involved when the distance is $<1 \mathrm{~mm}$ [17]. A clear but close margin is also considered to contribute to an adverse outcome. Thus, such margins should be quoted as positive or inadequate, and generally considered as an indication for adjuvant treatment [18]. Furthermore, margins are more often positive in oral cancer than elsewhere in the head and neck region, and patients with involved margins of $2 \mathrm{~mm}$ or closer have a higher incidence of local recurrence [19].

Depth of invasion (DOI) is a factor that currently modifies TNM. In the near future, it would be necessary to assess whether a DOI $>10 \mathrm{~mm}$ (pT3) should be managed with a combined treatment with EBRT and BT.

In a historical cohort of 277 patients with oral cavity carcinoma treated surgically, with a median follow-up of 36 months, positive or close margins were related with unfavorable results. The 5-year survival rate for $\geq 5 \mathrm{~mm}$ from the margin was $73 \%$, between 3 and $4 \mathrm{~mm}$ it was $69 \%$, for $\leq 2 \mathrm{~mm}$ was $62 \%$, and finally, for involved margins, it was 39\% [20].

Also, a 'single-operation' free margin was related with a better outcome compared to re-operated patients. Interestingly, a cut-off level of $5 \mathrm{~mm}$ was found to be decisive for the course of the disease, with significantly poorer survival rates and a trend towards more frequent local recurrence in deeper infiltrations [21].

On the other hand, the possible effects of mutilating surgery in a large tumor, which requires a large resection of the tongue, the organ-sparing approach emerges as a possibility, with radiotherapy as the mainstay, providing acceptable survival rates.

\section{Brachytherapy}

Brachytherapy as monotherapy is recommended for early-stage tongue carcinoma (T1N0 and T2N0 tumors $<4 \mathrm{~cm}$ ). For tumors $>3-4 \mathrm{~cm}$ or N1 lesions, although surgery is often preferred, BT can be delivered as a boost after 40-45 Gy of EBRT to the neck and oral cavity. In general, the local control rate is higher than $90 \%$ for $\mathrm{T} 1$ and T2N0 tumors treated with LDR-BT alone [5].

Leung et al. reported good outcomes for eight patients treated solely with HDR-ISBT. Five patients had T1N0 disease, and the remaining three were diagnosed with T2N0 disease. The median radiation dose was 60 Gy (10 fractions over 6 days). Mucositis for 6-20 weeks (median, 10 weeks) was observed in all patients. No local failure was evident after the median follow-up period of 26 months. One patient developed a grade 3 necrosis of the soft tissue and bone. They concluded that HDR remote afterloading technique is useful because it provides a local control rate of $100 \%$ with acceptable morbidity [22].

Umeda et al. reported the results of retrospective study performed among 180 patients with stage I/II 
tongue carcinoma, who were divided into three treatment groups: LDR $(n=78)$, HDR $(n=26)$, and surgery $(n=71)$. After salvage therapy, a final local cure was achieved in 71 patients (91\%) in the LDR group, $22(85 \%)$ in the HDR group, and $71(100 \%)$ in the surgery group [23].

Guinot et al. reported on 50 patients treated for oral cavity carcinoma with HDR-ISBT [8], with 42 cases with $\mathrm{T} 1 / 2 \mathrm{~N} 0$ tumors and 8 patients with T3 tumors. ISBT alone was administered to 17 (T1/2N0) patients $(34 \%)$, and 33 patients $(66 \%)$ received ISBT complementary to EBRT. A perioperative technique was performed in 14 patients. The actual DFS rates at three and five years were $81 \%$ and $74 \%$, respectively, with a median follow-up of 44 months. LC was maintained in all cases, in which HDR-BT was the only treatment. LC rates in the combined treatment group (EBRT + HDR-ISBT) were $80 \%$ and $69 \%$ at three and five years $(p=0.044)$, respectively. The authors concluded that HDR-ISBT is an effective method for treating tongue carcinoma in low-risk cases.

Ianovski et al. studied 41 patients $(74.6 \%)$ who received treatment with HDR-ISBT. OS at 3 and 5 years was $75.6 \%$ and $59.1 \%$, respectively, while CSS was $82.3 \%$ and $68.6 \%$, respectively. Cox regression analysis indicated that recurrence and survival outcomes were not associated with margin status or the use of a specific dose of HDR-ISBT. Acute and late toxicity, secondary to HDRISBT, was minimal [24].
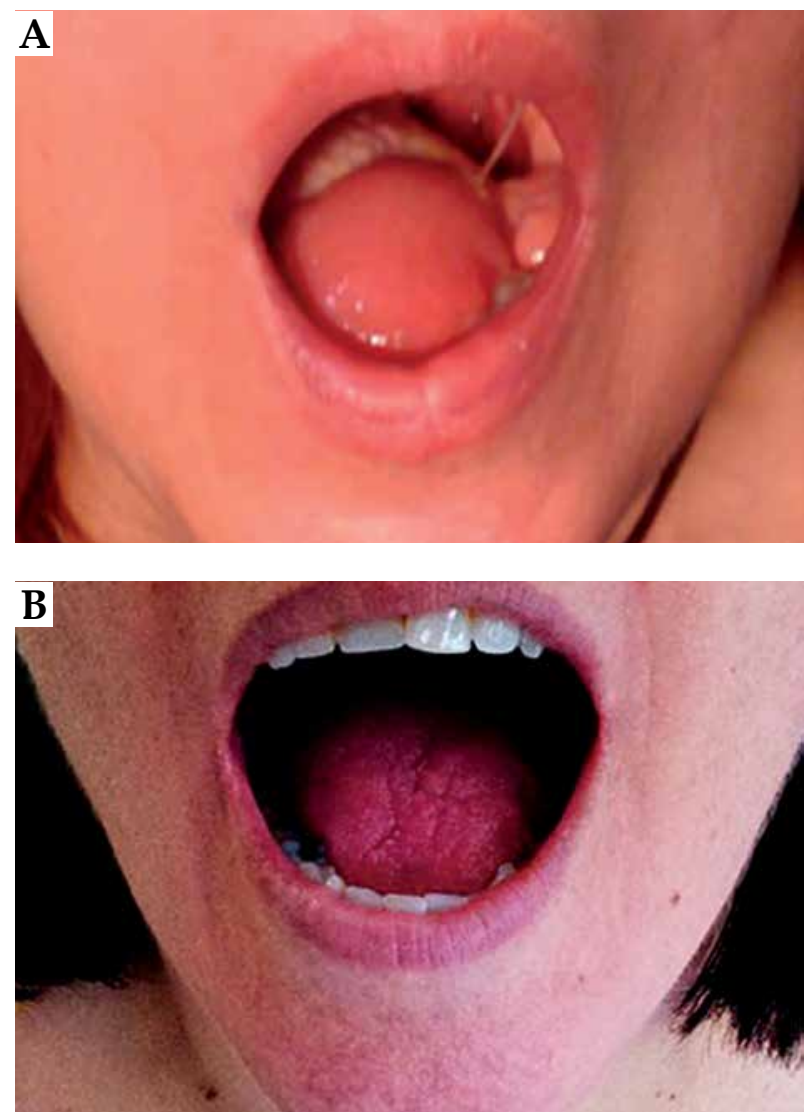

With this study, we confirm that a collaboration between a head and neck surgeon and radiation oncologist allows to optimize the advantages of tumor removal with early and accurate radiation. Thus, the presence of close or involved margins does not require a new surgical intervention, which would produce even greater alterations in function (Figure 5). BT ensured good local control in $75 \%$ of these cases. And if the margins are free, $95 \% \mathrm{LC}$ is achieved, similar or somewhat better than those of a large surgery, but using a more conservative surgery and better preserving the function. The cancer-specific survival achieved at 5 years was $88 \%$, and more than $60 \%$ of the patients finished the surgery and radiation treatment in 12 days.

\section{Conclusions}

Brachytherapy allows the administration of higher radiation doses with adjacent normal tissue sparing. In addition, it can be performed in the same surgical procedure, with an excellent view of CTV. In localized tumors, the most conservative surgeries are performed with very good LC, while the combined treatment with conservative surgery and ISBT has shown similar results compared to radical surgery or RT alone. Our results suggest that perioperative interstitial brachytherapy for squamous cell carcinoma of the tongue should be consid-
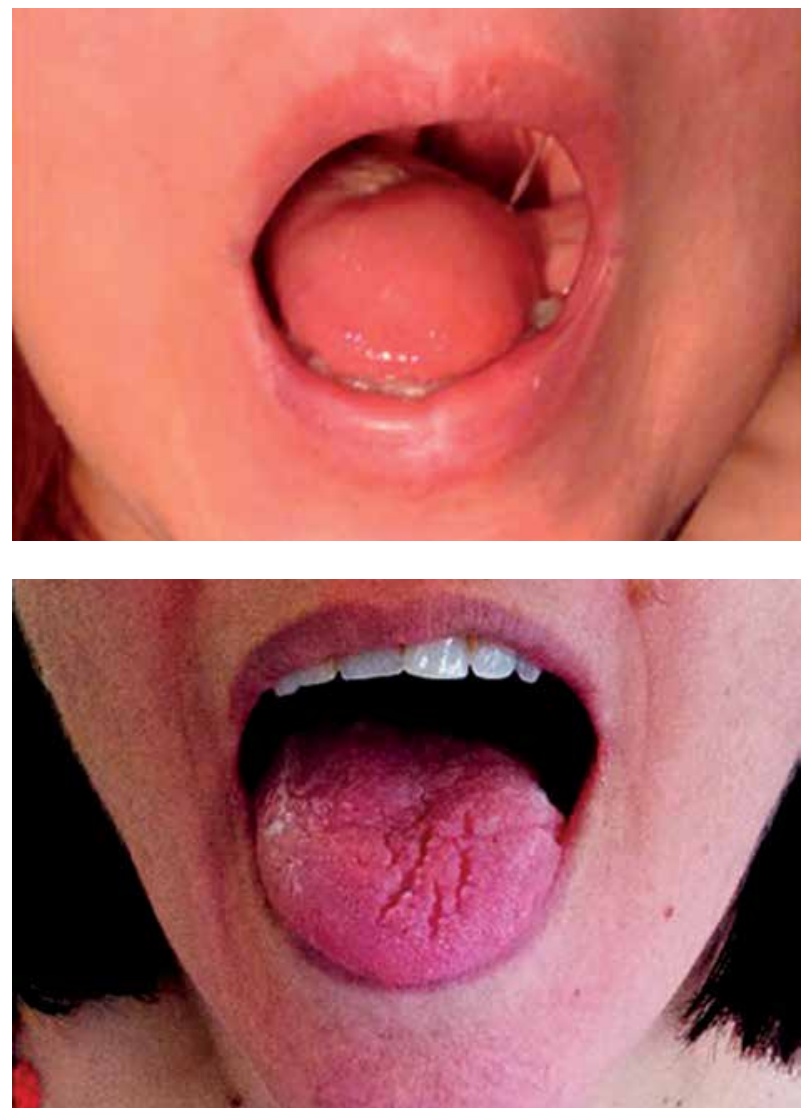

Fig. 5. Comparison of two patients, at rest and with maximum protrusion of the tongue. A) Hemiglossectomy with a graft. B) Partial glossectomy and perioperative BT (close margin, $10 \times 4$ Gy). Patient included in this study at 4 years of follow-up, with better function 
ered a valid option to perform a partial glossectomy of the macroscopic tumor with a small margin, to preserve better function and obtain good results, even if surgical margins are close or positive, without requiring a new surgery.

\section{Disclosure}

The authors report no conflict of interest.

\section{References}

1. Ganly I, Patel S, Shah J. Early-stage squamous cell cancer of the oral tongue - clinicopathologic features affecting outcome. Cancer 2012; 118: 101-111.

2. Preis M, Hadar T, Soudry TE et al. Early tongue carcinoma: analysis of failure. Head Neck 2012; 34: 418-421.

3. Hinerman RW, Mendenhall WM, Morris CG et al. Postoperative irradiation for squamous cell carcinoma of the oral cavity: 35-year experience. Head Neck 2004; 26: 984-994.

4. Iyer NG, Kim L, Nixon IJ et al. Outcome of patients with early T1 and T2 squamous cell carcinoma of the base of tongue managed by conventional surgery with adjuvant postoperative radiation. Head Neck 2013; 35: 999-1006.

5. Mazeron JJ, Ardiet JM, Haie-Meder C et al. GEC-ESTRO recommendations for brachytherapy for head and neck squamous cell carcinomas. Radiother Oncol 2009; 91: 150-156.

6. Kovacs G, Martinez-Monge R, Budrukkar A et al. GEC-ESTRO ACROP recommendations for head \& neck brachytherapy in squamous cell carcinomas: 1st update. Improvement by cross sectional imaging-based treatment planning and stepping source technology. Radiother Oncol 2017; 122: 248-254.

7. Goineau A, Piot B, Malard O et al. Postoperative interstitial brachytherapy for resectable squamous cell carcinoma of the tongue. Brachytherapy 2015; 14: 71-76.

8. Guinot JL, Santos MA, Tortajada MI et al. Efficacy of highdose-rate interstitial brachytherapy in patients with oral tongue carcinoma. Brachytherapy 2010; 9: 227-234.

9. Santos MA, Guinot JL, Tortajada MI et al. High-dose-rate interstitial brachytherapy boost in inoperable locally advanced tongue carcinoma. Brachytherapy 2017; 16: 1213-1218.

10. Yuen PW, Lam KY, Chan AC et al. Clinicopathological analysis of local spread of carcinoma of the tongue. Am J Surg 1998; 175: 242-244

11. Scholl P, Byers RM, Batsakis JG et al. Microscopic cut-through of cancer in the surgical treatment of squamous carcinoma of the tongue. Prognostic and therapeutic implications. Am J Surg 1986; 152: 354-360.

12. Looser KG, Shah JP, Strong EW et al. The significance of "positive" margins in surgically resected epidermoid carcinomas. Head Neck Surg 1978; 1: 107-111.

13. Loree TR, Strong EW. Significance of positive margins in oral cavity squamous carcinoma. Am J Surg 1990; 160: 410-414.

14. Sutton DN, Brown JS, Rogers SN et al. The prognostic implications of the surgical margin in oral squamous cell carcinoma. Int J Oral Maxillofac Surg 2003; 32: 30-34.

15. Slootweg PJ, Hordijk GJ, Schade $Y$ et al. Treatment failure and margin status in head and neck cancer. A critical view on the potential value of molecular pathology. Oral Oncol 2002; 38: 500-503.

16. Chandu A, Adams G, Smith AC. Factors affecting survival in patients with oral cancer: an Australian perspective. Int J Oral Maxillofac Surg 2005; 34: 514-520.

17. Woolgar JA, Triantafyllou A. A histopathological appraisal of surgical margins in oral and oropharyngeal cancer resection specimens. Oral Oncol 2005; 41: 1034-1043.
18. Zanoni DK, Migliacci JC, Xu B et al. A proposal to redefine close surgical margins in squamous cell carcinoma of the oral tongue. JAMA Otolaryngol Head Neck Surg 2017; 143: 555-560.

19. Jones AS, Bin Hanafi, Z, Nadapalan V et al. Do positive resection margins after ablative surgery for head and neck cancer adversely affect prognosis? A study of 352 patients with recurrent carcinoma following radiotherapy treated by salvage surgery. Br J Cancer 1996; 74: 128-132.

20. Nason RW, Binahmed A, Pathak KA et al. What is the adequate margin of surgical resection in oral cancer? Oral Surg Oral Med Oral Pathol Oral Radiol Endod 2009; 107: 625-629.

21. Ganly I, Goldstein D, Carlson DL et al. Long-term regional control and survival in patients with "low-risk", early-stage oral tongue cancer managed by partial glossectomy and neck dissection without postoperative radiation: the importance of tumour thickness. Cancer 2013; 119: 1168-1176.

22. Leung TW, Wong VY, Wong CM et al. High dose rate brachytherapy for carcinoma of the oral tongue. Int J Radiat Oncol Biol Phys 1997; 39: 1113-1120.

23. Umeda M, Komatsubara H, Ojima Y et al. A comparison of brachytherapy and surgery for the treatment of stage I-II squamous cell carcinoma of the tongue. Int J Oral Maxillofac Surg 2005; 34: 739-744.

24. Ianovski I, Mlynarek AM, Black MJ et al. The role of brachytherapy for margin control in oral tongue squamous cell carcinoma. J Otolaryngol Head Neck Surg 2020; 49: 74. 